\title{
EL ARBITRAJE Y SU UTILIZACION POR LAS EMPRESAS DE TACNA
}

\author{
Responsable : Abog. Démber Fernández Hernani Aragón. \\ Miembros : Abog. Juan Ramón Lira Loayza. \\ Abog. José Antonio Cárdenas Ticona.
}

\section{RESUMEN}

Se estudiaron las principales causas por las cuales las empresas de la ciudad de Tacna no utilizan el arbitraje como un medio alternativo de resolución de conflictos (MARC's). Los resultados obtenidos nos indican que $70 \%$ de las empresas consultadas en el presente trabajo desconoce el arbitraje y sus ventajas, especialmente las pequeñas y microempresas, siendo esta la principal causa de su no utilización. Otras causas por las que no se utiliza el arbitraje son porque no existen Centros de Arbitraje en Tacna (10\%); porque no lo consideran como un medio efectivo para la solución de los conflictos (10\%), y porque hasta ahora nunca han tenido la necesidad de recurrir a un medio de solución de conflictos (10\%). Asimismo, luego de explicar algunas ventajas del arbitraje se pudo verificar que existe disposición de las empresas consultadas de utilizar el arbitraje por considerarla principalmente como un medio más rápido de solución de sus conflictos, siempre y cuando sus costos no sean muyelevados.

\begin{abstract}
It has been studied the principals causes for finding the reasons why Tacna's companies do not use "Arbitration" as an alternative mean for solving their conflicts (MARC'S). The results show usthat the $70 \%$ of the polled companies (in the present work) don't know about "Arbitration" and its advantages, specially the small companies and microcompanies, that's why they do not use it. There are other reasons for not using "Arbitration" like: there are no Arbitration Centers in Tacna (10\%), another reason it's because they do not consider it as an effective mean for solving their problems (10\%), and because until now they haven't felt the need for finding the mean to solve their conflicts (10\%). Otherwise, after giving them an explanation of arbitration's advantages; it has been confirmed that there exits a possibility from the tested companies for using Arbitration, because they consider it as the principal and easier mean for solving their problems, but at the same they said it will depend on its cost price.
\end{abstract}

\section{INTRODUCCIÓN}

Haciendo un recorrido por la historia tenemos que los origenes del arbitraje se remontan a los pueblos comerciantes de la antigüedad, en donde se narran litigios existentes entre mercaderes griegos y fenicios que acudian al arbitraje con la finalidad de solucionar sus conflictos.

Demóstenes refiere que en Atenas, Solón expidió leyes reconociendo el arbitraje,cuyo laudo no era susceptible de recursos. Es asi como se puede apreciar que el arbitraje, a través de la historia, ha venido siendo utilizado por los comerciantes para la solución de sus conflictos de manera más rápida y eficaz. En la actualidad, muchos entendidos en la materia consideran al arbitraje como un medio eficaz para la solución pacífica de los conflictos existentes entre empresarios y entre estos con particulares, el cual se caracteriza por ser rápido, barato y tener seguridad juridica; así como evita estar envueltos en largos y costosos procesos judiciales, a partir de la aceptación que hacen las partes de someter su conflicto a uno o varios árbitros, quienes actúan a modo de juez y que luego de escucharlas, tomarán una decisión (laudo arbitral) que debe ser obligatoriamente aceptada.

Pese a las ventajas con las que se presenta el arbitraje, podemos apreciar que este no es empleado por las empresas de la ciudad de Tacna, quienes principalmente recurren al Poder Judicial para la solución de sus conflictos, situación que se debe al poco conocimiento de esta institución, asi como a la inexistencia de centros de arbitraje que lleven adelante el procedimiento arbitral, el cual cuente con personal capacitado para actuar como árbitros, tal como sí ocurre en otros lugares del pais como Lima y Arequipa.

En un sentido práctico, lo que se ha pretendido con la presente investigación es encontrar las principales causas por las cuales el arbitraje no viene siendo utilizando como un MARC's en la ciudad de Tacna, lo cual servirá para reajustar políticas de acciones concretas a los efectos de que se pueda revertir en algo dicha situación.

\section{OBJETIVO}

Establecer las principales causas por las que las empresas de la ciudad de Tacna no utilizan el arbitraje como medio alternativo para la solución de sus conflictos.

\section{MATERIAL Y MÉTODOS}

\section{1) Lugar de Ejecución}

El presente trabajo se ha realizado en la ciudad de Tacna, con la finalidad de conocer las causas por las cuales las empresas de nuestra ciudad no utilizan el arbitraje como un medio alternativo de solución de sus conflictos y comprobar si esto se debe a su desconocimiento o a otras causas.

\section{2) Diseño de la Investigación}

En el presente trabajo se utilizó un estudio con diseño descriptivo, explicativo y aplicativo. 


\section{3) Muestras delEstudio}

Las muestras de estudio fueron recogidas de 20 empresas formalmente constituidas (05 grandes y medianas empresas y 15 pequeñas y microempresas) de la ciudad de Tacna.

\section{4) Técnicas y Métodos de Trabajo}

Es un estudio de tipo descriptivo-explicativo. La técnica de recolección de datos fue el análisis documental y se utilizó información proporcionada por la Cámara de Comercio de Lima y de Arequipa, entre otras.

\section{5) Recolección de Datos}

La encuesta:

\author{
Entrevista y cuestionario \\ La observación. \\ Libros e informes \\ Estadisticos.
}

\section{DISCUSIÓN SOBRE EL TEMA}

\section{1) Medios Alternativos de Resolucion de Conflictos (marc's)}

Los medios alternativos de solución de conflictos son aquellos mecanismos que buscan dar una salida o solución extrajudicial a la pugna de intereses y/o posiciones que se presentan entre las partes que afrontan un conflicto. Se caracterizan por ser: voluntarios, privatistas, complementarios al Poder Judicial.

\section{La doctrina clasifica a los MARC's en:}

- Autocompositivos: Son aquellos donde las mismas partes de mutuo acuerdo dan solución al conflicto, aqui tenemos a la negociación, la mediación y la conciliación; y

- Heterocompositivos: Son aquellos donde es un tercero, ajeno a las partes, el que resuelve el conflicto, aqui ubicamos al arbitraje. En este sentido, el arbitraje es un MARC's Heterocompositivo, pues el que va a resolver el conflicto es un tercero elegido por las partes y llamado árbitro.

\section{2) ElArbitraje, Importancia y Ventajas}

La palabra arbitraje deriva del verbo ARBITRAR que significa DECIDIR,JUZGAR, DISCERNIR, ENJUICIAR OFORMAR JUICIO.

ZAIRA OSORIO RUIZ señala que "se ha definido al arbitraje como una técnica orientada a solucionar los conflictos entre dos o más personas, por una o varias otras personas (el árbitro o los árbitros), quienes poseen poderes de una convención privada y actúan según a esta convención sin estar investidos de esta misión por el Estado"
Para GUILLERMO LOMAN LUCA DE TENA el arbitraje es la institución que regula el acuerdo de voluntades por el cual dos o más partes deciden someter a uno o más terceros, que acepten el encargo, la resolución de un cierto conflicto de derecho privado respecto del cual dichas partes en capacidad de disposición, obligándose previamente a no llevar la controversia a los tribunales ordinarios sin el previo fallo arbitral, el cual deberá expedirse con arreglo a ciertas formalidades. Para CABANELLAS es toda decisión dictada por un tercero, con autoridad para ello en una cuestión 0 asunto que las partes han sometida su decisión y que tienen que cumplirse obligatoriamente.

Según KROTOSCHIN, el arbitraje suple el entendimiento directo de las partes y reemplaza el acuerdo entre ellas, por una decisión del conflicto que proviene de un tercero llamado árbitro.

La Ley N² 26572 (Ley General de Arbitraje) define al arbitraje como un método de solución de controversias en virtud del cual las partes acuerdan (convenio arbitral) someter la solución de determinados conflictos que hayan surgido o puedan surgir entre ellas respecto de una determinada relación jurídica a la decisión (laudo arbitral) de uno o varios terceros (árbitros). La forma de nombramiento de los terceros también es acordada por las partes.

Una excepción recogida en la Ley General de Arbitraje, al arbitraje convencional, es el arbitraje testamentario y el estatutario.

En el fondo del arbitraje existe un pacto o convenio entre los litigantes en el sentido de que someterán sus voluntades a la convicción y al pronunciamiento de tercero, con el compromiso de cumplir lo que por él se decida.

La importancia del Arbitraje radica en el valor que la ley le da a las decisiones tomadas por los árbitros al resolver un conflicto a través de la expedición de los LaudosArbitrales.

En este sentido, nuestra legislación da a dichos Laudos el valor equivalente a una Sentencia, tal como lo prescribe el Artículo 83 de la Ley 26572 (Ley General de Arbitraje) al establecer lo siguiente: "El aludo Arbitral consentido o ejecutoriado tiene valor equivalente a una sentencia eficaz y es eficaz y de obligatorio cumplimiento desde sunotificaciónalas partes. Si lo ordenado en el Laudo no se cumple por la parte o partes a quienes corresponde hacerlo el interesado podrá solicitar su ejecución forzada ante el juez especializado en lo civil del lugar de la sede del arbitraje que corresponde".

Asimismo, el Código Procesal Civil del Perú da a los laudos arbitrales la calidad de Titulo de Ejecución, al señalar en el Art. 713 lo siguiente: "Artículo 713: Son Titulos de Ejecución: 1. Resoluciones judiciales firmes. 2. Laudos arbitrales firmes. 3 . Los que la ley señale (Ley 26872 - Conciliación Extrajudicial - Acta de conciliación)". 
La ventaja que más se destaca del arbitraje es la celeridad procesal, que se traduce en un significativo ahorro de tiempo y dinero para las partes intervinientes. No obstante, este medio heterocompositivo cuenta con otras ventajas que le permiten convertirse en un instrumento eficaz de solución de diferencias entre empresas o particulares. Entre las ventajas más importantes destacan: la flexibilidad, privacidad, simplicidad e imparcialidad.

\section{3) ElConvenio Arbitral}

Fernando Cantuarias Salaverry y Manuel Diego Aramburú Yzaga definen el convenio arbitral asi: "El término convenio arbitral no hace más que reconocer que la suscripción de un acuerdo de arbitraje celebrado tanto antes como después de que surja la controversia, es suficiente para que las partes puedan recurrir al arbitraje directamente sin necesidad de otorgar ningún otro contrato".

Roque J. Caivano define al Convenio Arbitral como "el acuerdo de voluntades entre las partes", el cual tiene como consecuencia "la exclusión de los órganos estatales de administración de justicia para la resolución de uno o más conflictos determinados".

El articulo $4^{\circ}$ de la Ley General de Arbitraje contiene la definición de Convenio Arbitral: "El convenio arbitral es el acuerdo por el que las partes deciden someter a arbitraje las controversias que hayan surgido o puedan surgir entre ellas respecto de una determinada relación jurídica contractual o no contractual, sean o no materia de un proceso judicial".

La importancia del convenio arbitral radica en que a través de él las partes deciden someter a arbitraje las controversias que hayan surgido o puedan surgir entre ellas respecto a una determinada relación juridica contractual o no contractual, sea o no materia de un proceso judicial; es asi como una forma de propiciar la utilización del arbitraje sería que la empresas de la ciudad de Tacna incluyan dentro de sus contratos las cláusula pertinente que contenga el convenio arbitral.

\section{4) Instituciones Arbitrales o Centros de Arbitraje en el Peru y en Tacna}

Según la Ley General de Arbitraje, las partes pueden elegir directamente al tercero para que realice las funciones de árbitro (Arbitraje Ad-Hoc), pero también da la posibilidad de que puedan recurrir a instituciones dedicas y especializadas en dichos temas (Arbitraje Institucional), las cuales son las que nombran de un listado al árbitro u árbitros.

Estas instituciones arbitrales que toman el nombre de Centros de Arbitraje se encuentran reguladas en el Art. 8 de la Ley General de Arbitraje al establecer lo siguiente: "La organización y desarrollo del arbitraje puede ser encomendada a una institución Arbitral, la cual necesariamente deberá constituirse como Persona Juridica. En tal caso, la institución arbitral estará facultada para nombrar a los árbitros, asi como para establecer el procedimiento y las demás reglas a las que se someterá".
En el Perú, los Centros de Arbitraje se han desarrollado a través de la iniciativa privada, asi como con el auspicio de las Cámaras de Comercio. Es así que entre los principales Centros de Arbitraje a nivel nacional tenemos a los siguientes: El Centro de Arbitraje de la Cámara de Comercio de Lima, el Centro de Arbitraje de la Cámara de Comercio de Arequipa y el Centro de Arbitraje de la Cámara de Comercio Americana AMCHAM-PERU.

Por otro lado, según la información proporcionada por la Cámara de Comercio de Tacna, en nuestra ciudad no existe un Centro de Arbitraje auspiciado por dicha Cámara de Comercio, ni tampoco otros Centros de Arbitraje formalmente constituidos con el referido fin, lo cual hace que en nuestra ciudad las personas que desean optar por el arbitraje institucional no tengan dónde acudir.

Como toda entidad que brinda un servicio, en este caso de resolver un conflicto, los Centros de Arbitraje tienen derecho a una retribución que sirva para cubrir principalmente sus gastos administrativos, pago de honorarios a los árbitros, entre otros. Es por ello que al recurrir al arbitraje se tiene que efectuar el pago de aranceles y tasa, los cuales deben ser asumidos por las partes.

Dentro de algunos de los pagos que se tienen que efectuar en el Centro de Arbitraje de la Cámara de Comercio de Lima, se tiene por presentación de solicitud US\$ 120.00 , por nombramiento de árbitro US\$250.00, por copia certificada US\$1.00 (hoja). De otro lado, en el Centro de Arbitraje AMCHAM PERU la tasa administrativa es de US\$500.00 cuando la cuantia es hasta US\$10,000.00, en tanto que los honorarios por tres árbitros es de US\$1,500.00.

Teniendo en cuenta estos costos, las empresas consultadas las consideraron un tanto elevadas, sobre todo los micro y pequeños empresarios, quienes estarian dispuestos a someter la solución de sus conflictos al arbitraje, pero considerando costos no muy elevados.

\section{RESULTADO}

La hipótesis planteada en la presente investigación ha sido: "La falta de un adecuado conocimiento del arbitraje (legislación, ventajas, inclusión del compromiso arbitral como una cláusula en el contrato, etc) y la no existencia de centros de arbitraje ha motivado que las empresas de la ciudad de Tacna no lo utilicen como un medio alternativo para la solución de sus conflictos."

La contrastación y comprobación de esa hipótesis ha requerido el estudio de las variables dentro de las cuales se realizó el estudio teórico documentado del arbitraje, de donde se desprende que éste es un medio alternativo de solución de conflictos que puede ser usado por las empresas de la ciudad de Tacna.

Es asi que el arbitraje tendria una utilización práctica en la región si se hace una adecuada difusión del mismo y si se constituyen centros de arbitraje con personal debidamente capacitado. 
Esto es corroborado con los resultados del tratamiento estadisticos de los datos obtenidos de la consulta efectuada a 20 empresas (05 grandes y medianas empresas; y 15 pequeñas y micro empresas) y que figuran en las conclusiones del presente trabajo

\section{CONCLUSIONES}

1. El arbitraje se constituye en un instrumento eficaz para la solución pacífica de conflictos entre empresarios y entre estos con particulares. Se caracteriza por ser rápido y por tener seguridad juridica, evitando entrar en largos y costosos procesos judiciales. Sin embargo, $70 \%$ de las empresas consultadas en el presente trabajo de investigación desconocen el arbitraje y sus ventajas, principalmente las pequeñas y microempresas.

2. Pese a las ventajas del arbitraje, se ha podido comprobar que este no es empleado por las empresas de la ciudad de Tacna, las cuales sólo recurren al Poder Judicial para la solución de sus conflictos.

3. Las principales causas por las que los empresarios consultados no han recurrido al arbitraje son por el desconocimiento de la existencia del arbitraje como un medio alternativo de solucionar sus conflictos $(70 \%)$, por la no existencia de Centros de Arbitraje en Tacna (10\%), por considerar que el arbitraje no es un medio efectivo de solución de los conflictos (10\%), porque nunca ha tenido la necesidad de recurrir a un medio de solución de conflictos (10\%). Existe disposición en las empresas de la ciudad de Tacna para utilizar el arbitraje por considerarlo principalmente como un medio más rápido de solución de sus conflictos.

4. En caso de recurrir al arbitraje, las empresas tienen preferencia por escoger como árbitro a un Centro de Arbitraje (Arbitraje Institucional) y no a una persona particular (Arbitraje Ad Hoc); sin embargo, en nuestra ciudad no existen centros de arbitraje, ni siquiera auspiciados por la Cámara de Comercio de Tacna.

5. Las tasas y aranceles que cobran los Centros de Arbitraje que funcionan en Lima y en Arequipa son un tanto elevadas para las posibilidades de las pequeñas y microempresas, las cuales estaría dispuestas a pagar, pero una cantidad razonable dependiendo del caso.

6. La mayoría de empresas desconocen que el sometimiento al arbitraje es a través de la firma de un convenio arbitral entre las partes; sin embargo, dependiendo del caso estarian dispuestas a incluirla dentro de sus contratos.

\section{RECOMENDACIONES}

De los resultados obtenidos en este trabajo de investigación, nos permitimos recomendar lo siguiente:

Proporcionar a los empresarios de la ciudad de Tacna una mayor información acerca del arbitraje y sus ventajas como medio alternativo de solución de sus conflictos, ello a través de seminarios, talleres, cursos y material informativo como el que se anexa al presente trabajo de investigación.

Estudiar la posibilidad de constituir un Centro de Arbitraje de la Universidad Nacional Jorge Basadre Ghomann a través de la Facultad de Letras y Ciencias Juridicas, a cargo de la Escuela de Derecho, ya que cuenta con infraestructura necesaria (Sala de Audiencias) y profesores especialistas en distintas ramas del derecho quienes pueden actuar como árbitros (unipersonales o colegiados), con lo cual se estaria logrando uno de los fines de toda universidad, que es la proyección social.

Celebrar un Convenio Interinstitucional entre la Universidad Nacional Jorge Basadre Ghomann a través de la Facultad de Letras y Ciencias Jurídicas y la Cámara de Comercio de Tacna con el propósito de efectuar una adecuada difusión del arbitraje entre los miembros de dicho gremio comercial e incluir en los contratos que estos celebren el sometimiento al arbitraje.

\section{BIBLIOGRAFIA}

Osorio Ruiz, Zaida. Contratos Comerciales Empresariales yel Arbitraje. Edición IDEMSA Junio 2002.

Roque J., Caivano, Negociación, Conciliación y Arbitraje, Editado porAPENAC.

PINKAS FLINT, B. La Negociación Empresaria. Ediciones Justo Valenzuela EIRL,. Lima, 1993.

Ley General De Arbitraje, Ley N ${ }^{\circ} 26572$, Juristas Editores, Lima, 2006.

Guasp, Jaime. El Arbitraje en el Derecho Español, Editorial Bosch, Barcelona, 1956

Lorca Navarrete, Antonio. Derecho de Arbitraje Interno e Internacional, Editorial Tecnos, Madrid, 1989.

Lorca Navarrete, Antonio. Comentarios a Ley de Arbitraje, Editorial Dykinson, Madrid, 1991.

Devis Echandia, Hernando. La Situación del Arbitraje en Colombia. Editorial Temis, Bogotá. 1982.

Chillón Medina, José María Y Merino Merchán, José Fernando. Tratado de Arbitraje Privado Interno e Internacional. Editorial Civitas S.A., Madrid, 1978.

Monroy Cabra, Marco Gerardo. Arbitraje Comercial, Nacional e Internacional. $2^{\circ}$ da. Edición. Editorial Legis, Santa Fé de Bogotá, 1998. 